\title{
Optimization of Capactive Microphone and Pressure Sensor Performance by Capacitor-electrode Shaping
}

\author{
J. A. VOORTHUYZEN, A. J. SPRENKELS, A. G. H. VAN DER DONK, P. R. SCHEEPER and P. BERGVELD \\ University of Twente, P.O. Box 217, 7500 AE Enchede (The Netherlands)
}

\begin{abstract}
In many designs of capacitive microphones or pressure sensors the electrode size is chosen to be equal to the diaphragm size. In this paper it will be discussed whether an electrode size or shape that differs from that of the diaphragm is attractive for obtaining a maximum value for the sensor sensitivity and the signal-to-noise ratio. A theoretical analysis will be given for circular diaphragms and electrodes, from which it can be shown that for maximum sensitivity the electrode should be located at the centre of the diaphragm, with a radius depending on the value of the amplifier input capacitance.
\end{abstract}

\section{Introduction}

The application of capacitive structures in pressure sensors and microphones has been successfully explored for several decades. A recent example is the silicon-based electret microphone, with a sensitivity of $20 \mathrm{mV} / \mathrm{Pa}$, a dynamic range of $95 \mathrm{~dB}$ and a lower detection level of $400 \mu \mathrm{Pa}$ [1].

A capacitive microphone or pressure sensor consists of a rigid backplate, an air gap and a diaphragm which is exposed to the (sound) pressure to be detected. To realize a capacitor, the diaphragm and backplate are provided with conducting electrodes.

Characterization and optimization of tranducer performance has been a main topic in various recent papers on capacitive sensors for the mechanical domain [1-9], taking into account diaphragm thickness, lateral dimensions, etc. However, in these papers hardly

$0924-4247 / 91 / \$ 3.50$ any attention is paid to the size of the conductive electrodes, which determines the sensor capacitance. It will be obvious that there is no need to consider a redesign of the electrode shape if the diaphragm material as well as the support are conductive [2-5]. However, even in sensors with an insulating diaphragm or substrate, it is tacitly assumed that the optimum electrode shape corresponds to a complete metallization of the diaphragm [6-9].

In this paper it will be shown that an electrode size or shape not identical to that of the diaphragm may be very attractive.

\section{Theory of Pressure-sensitive Capacitors}

The theoretical description is focused on a circular diaphragm with flexural rigidity $D$, as determined by the material properties and diaphragm dimensions. If the diaphragm outer radius is written as $a$ and the uniform pressure difference across it as $P$, the deflection $w$ can be written as

$w(\gamma)=w_{0}\left(1-\gamma^{2}\right)^{2}$

with $\gamma$ the radial distance, normalized to the outer radius $a$ and

$w_{0}=P a^{4} /(64 D)$

assuming the diaphragm to operate in the linear region, its edge to be clamped and ignoring the influence of lateral stress in the diaphragm [10].

The diaphragm is assumed to be mounted on a supporting structure, enclosing an air gap as drawn in Fig. 1. On top of the insulating diaphragm a conductive electrode with

(C) Elsevier Sequoia/Printed in The Netherlands 


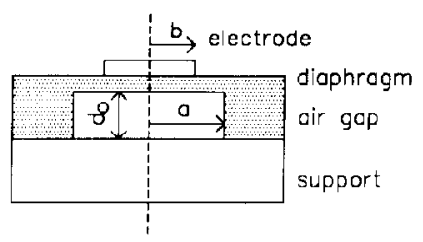

Fig. 1. Schematic drawing of a pressure-sensitive capacitor.

radius $b$ is deposited. The support is assumed to also be conductive, so that the value of the capacitor thus realized is determined by the radius of the electrode on top of the diaphragm and its distance from the support. Neglecting the diaphragm thickness, the zeropressure electrode distance equals the air gap distance $d_{0}$, as shown in Fig. 1 .

Introducing $\lambda=b / a$ as the normalized electrode radius, the capacitance $C$ can be written as

$C=2 \pi \epsilon_{0} a^{2} \int_{0}^{\lambda} \frac{\gamma \mathrm{d} \gamma}{d_{0}-w(\gamma)}$

with $\epsilon_{0}$ the dielectric constant of air. Using eqns. (1) and (3), the following analytical expression can be found:

$C=\frac{\pi \epsilon_{0} a^{2}}{2 d_{0} \zeta} \ln \left[\frac{1+\zeta^{2} \lambda^{2}-\zeta^{2}+\zeta \lambda^{2}}{1+\zeta^{2} \lambda^{2}-\zeta^{2}-\zeta \lambda^{2}}\right]$

with $\zeta^{2}=w_{0} / d_{0}$.

In a practical design the mechanical and geometrical parameters of the capacitive structure will be chosen in such a way that $\zeta^{2}=w_{0} / d_{0} \ll 1$ in the whole pressure region to be monitored. It will also be clear that $0 \leqslant \lambda \leqslant 1$.

Approximation of eqn. (4) by means of a Taylor series expansion yields

$C=C_{\mathrm{m}} \lambda^{2}\left[1+\frac{w_{0}}{d_{0}}\left(1-\lambda^{2}+\frac{\lambda^{4}}{3}\right)\right]$

with $C_{\mathrm{m}}=\pi \epsilon_{0} a^{2} / d_{0}$, the maximum zero-pressure capacitance that can be realized by a complete metallization of the diaphragm, and $C_{\mathrm{m}} \lambda^{2}$ the actual zero-pressure capacitance. Combination of eqns. (2) and (5) results in an expression that relates the applied pressure $P$ and the resulting capacitance $C$ :

$C=C_{\mathrm{m}} \lambda^{7}+K_{\mathrm{p}} C_{\mathrm{m}}\left(\lambda^{7}-\lambda^{4}+\frac{\lambda^{6}}{3}\right) P$

with $K_{\mathrm{p}}=a^{4} /\left(64 D d_{0}\right)$. The constants $C_{\mathrm{m}}$ and $K_{\mathrm{p}}$ are exclusively determined by the geometry of the capacitive structure as well as the mechanical properties of the diaphragm material.

In most sensor designs the whole diaphragm is metallized, thus $\lambda=1$. It is easy to see from eqn. (6) that this corresponds to the situation where the capacitance change $\delta C$ due to an applied pressure $\delta P$ is maximal. However, it will be shown below that the maximum signal and signal-to-noise ratio require values of $\lambda$ other than one.

\section{Theory of Capacitance-to-voltage Conversion}

Different types of electronic circuits have been developed for the detection of small capacitances and capacitance changes in the case of sensors for the mechanical domain. Examples of circuit configurations recently applied are the current-controlled oscillator [11], the switched capacitor circuit [12], the electret-biased capacitor $[1,13]$ and the a.c.driven capacitive bridge circuit [14]. Despite their different ways of operation, these configurations all have in common that the pressure-sensitive capacitor is biased by means of a voltage or current source and is connected to a high-impedance preamplifier. It can be shown that the overall performance of the complete system is determined by the pressure-sensitive capacitor and the preamplifier, including its bias circuitry.

It is impossible to discuss all the different types of preamplifiers. Therefore only the configuration shown in Fig. 2 will be discussed, which consists of a d.c. bias voltage $V_{\mathrm{b}}$, a bias resistor $R_{\mathrm{b}}$, a pressure-sensitive capacitance $C$ and a MOSFET source follower, which is assumed to provide unity gain from gate to source. 


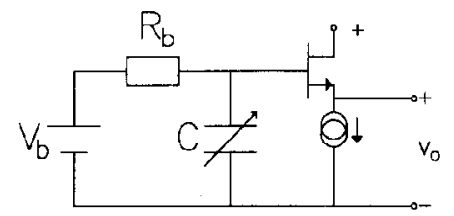

Fig. 2. Schematic drawing of a d.c.-biased pressuresensitive capacitor.

Considering Fig. 2 in more detail, it will be clear that if the capacitance $C$ is constant (no pressure change), the voltage across the capacitance $C$ is equal to the bias voltage $V_{\mathrm{b}}$. Assuming pressure changes to be fast compared to the time constant $C R_{\mathrm{b}}$, and the input capacitance of the MOSFET source follower to be zero, a capacitance change $\delta C$ results in a voltage change that is equal to $V_{\mathrm{b}} \delta C / C$. However, in practical situations the input capacitance of the MOSFET source follower will not be zero, but will have a finite value $C_{i}$, causing a capacitive attenuation, so that the actual small-signal output voltage $v_{\mathrm{o}}$ at the source due to a capacitance change can be written as

$v_{\mathrm{o}}=V_{\mathrm{b}} \frac{\delta C}{\left(C+C_{\mathrm{i}}\right)}$

\section{Optimization of Capacitor Electrode Geometry}

Now that a simple description of the pressure-to-capacitance and capacitance-tovoltage conversion has been presented, the optimized capacitor electrode geometry can be derived for different optimization criteria.

To avoid an over-complicated description, it will be assumed that the capacitance change $\delta C$ is small compared to the actual value $C$ of the capacitance, so that the value of $C$, as present in the denominator of eqn. (7), can be considered to be constant, independent of the applied pressure and equal to $C_{\mathrm{m}} \lambda^{2}$.

The sensitivity $S$ of the sensor with its preamplifier will simply be defined as the ratio of output voltage $v_{\mathrm{o}}$ and pressure $P$, thus $S=v_{\mathbf{o}} / P$.

\subsection{Optimum Output Signal}

Due to the fact that in most sensors the sensitivity is a critical point, a design criterion very often proposed is maximum output signal. Considering eqn. (7), this means that the ratio $\delta C /\left(C+C_{\mathrm{i}}\right)$ has to be maximized.

\subsubsection{Theory}

Combination of eqns. (6) and (7) yields

$S=\frac{V_{\mathrm{b}} K_{\mathrm{p}} C_{\mathrm{m}}\left(\lambda^{2}-\lambda^{4}+\frac{\lambda^{6}}{3}\right)}{C_{\mathrm{m}} \lambda^{2}+C_{\mathrm{i}}}$

Considering this equation, it is to be seen that for a zero value of the amplifier input capacitance $C_{i}$, maximum sensitivity is obtained if the normalized electrode radius $\lambda$ is very small. The maximum sensitivity is in that case equal to $V_{\mathrm{b}} K_{\mathrm{p}}$, which is the largest sensitivity that can ever be obtained with this capacitive structure.

If $C_{\mathrm{i}}$ is large compared to $C_{\mathrm{m}}$, a situation that may occur in careless designs, the maximum sensitivity appears to be $V_{\mathrm{b}} K_{\mathrm{p}} C_{\mathrm{m}} /\left(3 C_{\mathrm{i}}\right)$ and is obtained when $\lambda=1$, corresponding to a completely metallized diaphragm.

To calculate the optimal electrode radius for maximum sensitivity for values of $C_{\mathrm{i}}$ other than the limiting cases mentioned above, computer calculations of eqn. (8) have to be performed. The main results are shown in Figs. 3 and 4.

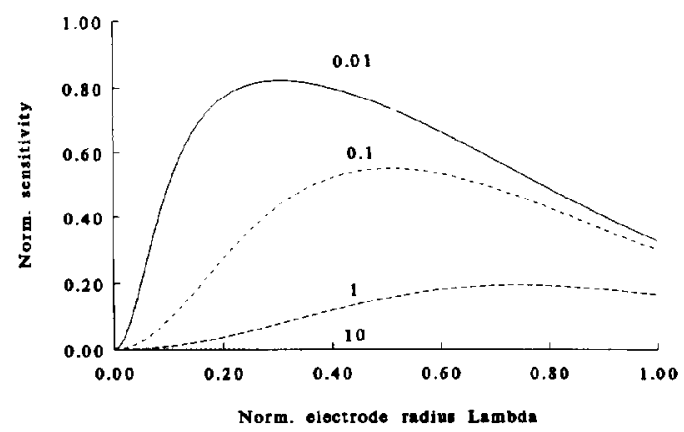

Fig. 3. Calculated normalized sensitivity as a function of the nomalized radius $\lambda$ with the capacitance ratio $C_{\mathrm{i}} / C_{\mathrm{m}}$ as a parameter. 


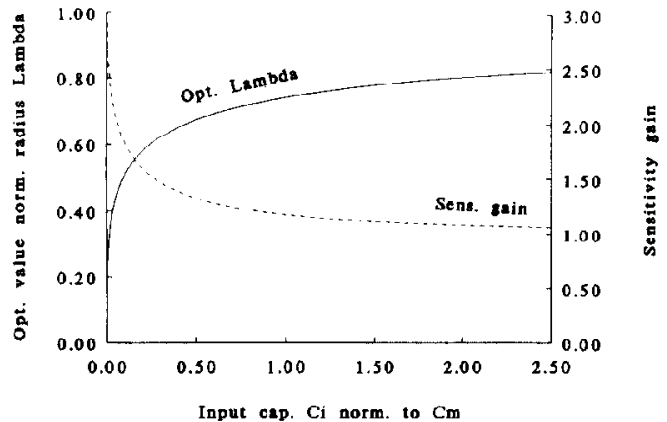

Fig. 4. Calculated optimum value of $\lambda$ (left axis, solid line) and sensitivity gain (right axis, dashed line) as a function of the capacitance ratio $C_{\mathrm{i}} / C_{111}$

In Fig. 3 the sensitivity, normalized to the maximum value $V_{\mathrm{b}} K_{\mathrm{p}}$, is drawn as a function of the normalized electrode radius $\lambda$, for different ratios $C_{\mathrm{i}} / C_{\mathrm{m}}$. If $C_{\mathrm{i}}=0.01 C_{\mathrm{m}}$, the maximum sensitivity is obtained for $\lambda=0.3$ and is equal to about $0.8 V_{\mathrm{b}} K_{\mathrm{p}}$. If for this $C_{\mathrm{i}} / C_{\mathrm{m}}$ value the complete diaphragm were metallized $(\lambda=1)$, the sensitivity would be equal to $0.33 V_{\mathrm{b}} K_{\mathrm{p}}$, which is 2.7 times lower than the maximum value. Larger values of $C_{\mathrm{i}} / C_{\mathrm{m}}$ require larger optimum values of $\lambda$, approaching $\lambda=1$ for values of $C_{\mathrm{i}}$ equal to or larger than $10 C_{m}$.

In Fig. 4 two curves are drawn. The solid line is related to the left $y$-axis and represents the optimum value of $\lambda$ for maximum sensitivity as a function of the ratio $C_{\mathrm{i}} / C_{\mathrm{m}}$. The dashed line corresponds to the right $y$-axis and represents the so-called sensitivity gain, defined as the maximum sensitivity achieved if $\lambda$ is chosen equal to its optimum value, normalized to the sensitivity obtained if $\lambda=1$. It can be observed that for very small values of $C_{\mathrm{i}} / C_{\mathrm{m}}$ the optimum value of $\lambda$ approaches zero. In this case the sensitivity is equal to $V_{\mathrm{b}} K_{\mathrm{p}}$. If, however, for this value of $C_{\mathrm{i}} / C_{\mathrm{m}} \lambda$ is chosen equal to its maximum value of one, the sensitivity would be equal to $0.33 V_{\mathrm{b}} K_{\mathrm{p}}$, which implies a sensitivity gain of three when $\lambda$ is chosen optimally. In the case $C_{\mathrm{i}} / C_{\mathrm{m}}=1$, the optimal value of $\lambda$ is 0.78 and the associated sensitivity gain 1.17.

Considering both Figures, it can be concluded that a significant sensitivity gain can be obtained in the case when the amplifier input capacitance $C_{\mathrm{i}}$ is small compared to $C_{\mathrm{m}}$ and the diaphragm is only partly metallized. However, if the amplifier input capacitance is large compared to $C_{\mathrm{m}}$, a complete metallization is required for maximum sensitivity.

\subsubsection{Experimental results}

In order to verify the theoretical description, the output signal of various electret microphones in combination with a sourcefollower MOSFET amplifier has been measured for different circular electrodes on top of square diaphragms. The deflection as a function of applied pressure for square diaphragms differs slightly from that for circular diaphragms, but this effect is assumed to be of minor importance, so that the theory of circular diaphragms can still be used.

The amplifier input capacitance $C_{\mathrm{i}}$ appeared to be about $1 \mathrm{pF}$, while the maximum value of the microphone capacitance $C_{\mathrm{m}}$ is about $3 \mathrm{pF}$ when the complete diaphragm is metallized. Using the theoretical results as shown in Fig. 4, this implies a maximum output signal for $\lambda=0.63$ and, as compared to the sensitivity for a completely metallized diaphragm, a sensitivity gain of 1.4 .

The experimental results of microphones for four different values of the normalized electrode radius are shown in Fig. 5. Each result is the average of three independently measured microphones. The vertical axis is

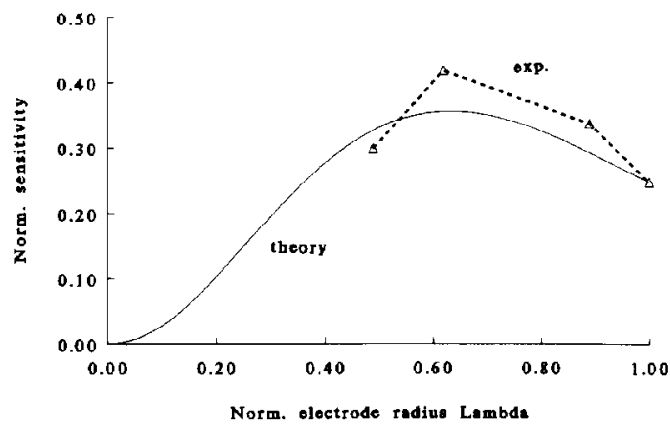

Fig. 5. Experimental results for the normalized sensitivity as a function of $\lambda$. Each point is the average sensitivity of three individual microphones. The solid line is the theoretical curve for $C_{\mathrm{i}}=0.33 C_{\mathrm{m}}$. 
the amplifier output signal scaled in such a way that for $\lambda=1$ theoretical and experimental results correspond to each other. The horizontal axis corresponds to the normalized electrode radius $\lambda$. The solid curve is calculated by means of eqn. (8) with $C_{\mathrm{m}}=3 C_{\mathrm{i}}$. It can be seen that experiments and theory are in good agreement and that microphones with $\lambda=0.63$ indeed exhibit the largest sensitivity, even more pronounced than expected from theory.

\subsection{Optimum Signal-to-noise Ratio}

In the previous Section the sensor performance has been optimized with respect to the maximum output signal. In practice, however, the overall performance of a sensor is determined by the signal-to-noise ratio $S N_{\mathrm{r}}$. Assuming sufficient amplification in the preamplifier, it will be appropriate to consider the signal-to-noise ratio at its output. In this paper only some general aspects will be considered. A detailed paper will be published elsewhere [15].

In summary it can be said that the signalto-noise ratio $S N_{r}$ of a capacitive pressure sensor, connected to a FET-type preamplifier, is mainly determined by the channel noise of the FET and the noise of the resistive bias element $R_{\mathrm{b}}$. Denoting the constants representing both noise terms as $N_{\mathrm{f}}$ and $N_{\mathrm{b}}$ respectively, the following approximated equation can be derived:

$\left(S N_{\mathrm{r}}\right)^{-2}=\frac{N_{\mathrm{f}}^{2}\left[\frac{C_{\mathrm{m}} \lambda^{2}+C_{\mathrm{j}}}{C_{\mathrm{m}} \lambda^{2}}\right]^{2}+N_{\mathrm{b}}^{2}\left[\frac{1}{\lambda^{2}}\right]^{2}}{\left[V_{\mathrm{b}} K_{\mathrm{p}}\left(1-\lambda^{2}+\lambda^{4} / 3\right) P\right]^{2}}$

where $C_{\mathrm{j}}$ is the physical input capacitance of the amplifier, as present if no local feedback in the preamplifier is used. Note that in Section 4.1. the signal amplitude has been written as a function of the input capacitance $C_{\mathrm{i}}$, which is the virtual or electronic input capacitance [15]. In the case of a source follower, it can be stated that $C_{\mathrm{j}} \geqslant C_{\mathrm{i}}$.

In order to reduce the complexity of eqn. (9), only the two extreme conditions in which the FET noise term $N_{\mathrm{f}}$ or the bias resistor noise term $N_{\mathrm{b}}$ dominates the signal-to-noise ratio will be considered.

Assuming the FET noise to dominate, it can easily be shown that the signal-to-noise ratio $S N_{\mathrm{r}}$ can be written as

$S N_{\mathrm{r}}=\frac{V_{\mathrm{b}} K_{\mathrm{p}} C_{\mathrm{m}}\left(\lambda^{2}-\lambda^{4}+\frac{\lambda^{6}}{3}\right)}{C_{\mathrm{m}} \lambda^{2}+C_{\mathrm{j}}} \frac{P}{N_{\mathrm{f}}}$

Comparing eqns. (8) and (10), it is seen that in this case the conditions for maximum output signal and maximum signal-to-noise ratio are almost identical. The only difference is that the expression for the output signal is determined by the virtual input capacitance $C_{i}$ and the expression for the signal-to-noise ratio by the physical input capacitance $C_{\mathrm{j}}$. The results shown in Figs. 3 and 4 can therefore also be used to determine the optimum electrode geometry for optimized signal-tonoise ratio.

If the bias resistor noise term $N_{\mathrm{b}}$ is dominating, eqn. (9) can be simplified to

$S N_{\mathrm{r}}=V_{\mathrm{b}} K_{\mathrm{p}}\left(\lambda^{2}-\lambda^{4}+\frac{\lambda^{6}}{3}\right) \frac{P}{N_{\mathrm{b}}}$

Considering this equation, the optimum value of $\lambda$ for a maximum value of the signal-tonoise ratio appears to be independent of the input capacitance of the preamplifier and to be equal to one. This means that if the bias resistor noise term is dominating, the maximum signal-to-noise ratio implies a completely metallized diaphragm.

If the relative contributions of both noise terms are of the same magnitude, the complete expression of eqn. (9) has to be used to calculate the optimum capacitor electrode geometry. The optimum value of $\lambda$ will then be in between the values correponding to the limiting cases described in eqns. (10) and (11).

\section{Discussion and Conclusions}

Using a quite simple theoretical description for the pressure-to-capacitance conversion of circular, stress-free diaphragms, as 
well as the capacitance-to-voltage conversion, a model has been obtained by which the performance of pressure sensors and microphones can be calculated.

It has been shown that when the pressuresensitive capacitance is connected to a preamplifier with small input capacitance, a sensitivity gain of about three can be obtained if only a small central part of the diaphragm is metallized. When the preamplifier input capacitance is large compared to the sensor capacitance, the maximum output signal is obtained by metallizing the diaphragm completely.

Taking into account the noise contribution of the preamplifier, it can be shown that the conditions for maximum signal-to-noise ratio depend, besides the influence of the amplifier input capacitance already discussed, on the relative contributions of the FET noise and the bias resistor noise. If the bias resistor noise dominates, the complete diaphragm should be metallized, while if the transistor noise dominates, a small circular metallization is preferably.

\section{References}

1 J. A. Voorthuyzen, P. Bergveld and A. J. Sprenkels, Semicondunctor-based electret sensors for sound and pressure, IEEE Trans. Electr. Insul., EI-24 (1989) 267-276.

2 Y. S. Lee and K. D. Wise, A batch-fabricated silicon capacitive pressure transducer with low temperature sensitivity, IEEE Trans. Electron Devices, $E D-29$ (1982) 42-48.

3 K. E. Petersen, Capacitive pressure transducer, U.S. Patent No. 4332000 (May 1982).
4 M. M. Farooqui and A. G. R. Evans, A polysilicondiaphragm-based pressure sensor technology, $J$. Phys. E: Sci. Instrum., 20 (1987) 1469-1471.

5 H.-L. Chau and K. D. Wise, An ultraminiature solid-state pressure sensor for a cardiovascular catheter, IEEE Trans. Electron Devices, ED-35 (1988) 2355-2362.

6 H.-L. Chau and K. D. Wise, Scaling limits in batchfabricated silicon pressure sensors, IEEE Trans. Electron Devices, ED-34 (1987) 850-858.

7 G. Blasquez, Y. Naciri, P. Blondel, N. Bem Moussa and $\mathbf{P}$. Pons, Static response of miniature capacitive pressure sensors with square or rectangular silicon diaphragm, Rev. Phys. Appl., 22 (1987) 505-510.

8 D. Hohm and G. Hess, A subminiature condenser microphone with silicon nitride membrane and silicon back plate, J. Acoust. Soc. Am., 85 (1989) 476-480.

9 G. Blasquez, P. Pons and A. Boukabache, Capabilities and limits of silicon pressure sensors, Sensors and Actuators, 17 (1989) 387-403.

10 S. P. Timoshenko and S. Woinowsky-Krieger, Theory of Plates and Shells, McGraw-Hill, London, 2nd edn., 1984, pp. 51-58.

11 M. J. S. Smith, L. Bowman and J. D. Meindl, Analysis, design, and performance of a capacitive pressure sensor IC, IEEE Trans. Biomed. Eng., BME-33 (1986) 163-174.

12 Y. E. Park and K. D. Wise, An MOS switchedcapacitor readout amplifier for capacitive pressure sensors, IEEE 1983 Custom Integrated Circuits Conf., May 1983, pp. 380-384.

13 A. J. Sprenkels, R. A. Groothengel, A. J. Verloop and P. Bergveld, Development of an electret microphone in silicon, Sensors and Actuators, 17 (1989) 509-512.

14 W. H. Ko, M.-H. Bao and Y.-D. Hong, A highsensitivity integrated-circuit capacitive pressure transducer, IEEE Trans. Electron Devices, ED-29, (1982) $48-56$.

15 A. G. H. van der Donk, J. A. Voorthuyzen and P. Bergveld, Optimal design of electret microphone MOSFET preamplifier, J. Acoust. Soc. Am., submitted for publication. 